\title{
Serum uric acid in US adolescents: distribution and relationship to demographic characteristics and cardiovascular risk factors
}

\author{
Ibrahim F. Shatat ${ }^{1}$, Rany T. Abdallah², David J. Sas ${ }^{1}$ and Susan M. Hailpern ${ }^{3}$
}

INTRODUCTION: Despite being associated with multiple disease processes and cardiovascular outcomes, uric acid (UA) reference ranges for adolescents are lacking. We sought to describe the distribution of UA and its relationship to demographic, clinical, socioeconomic, and dietary factors in US adolescents.

METHODS: A nationally representative subsample of 1,912 adolescents $13-18$ y of age, from the National Health and Nutrition Examination Survey (NHANES) for the years 20052008 representing 19,888,299 adolescents, was used for this study. Percentiles of the distribution of UA were estimated using quantile regression. Linear regression models examined the association of UA with demographic, socioeconomic, and dietary factors.

RESULTS: The mean UA level was $5.14 \pm 1.45 \mathrm{mg} / \mathrm{dl}$. It increased with increasing age and was higher in non-Hispanic whites, male sex, those with higher BMI z-scores, and those with higher systolic blood pressure (BP). In fully adjusted linear regression models, sex, age, race, and BMI were independent determinants of higher $U A$.

DISCUSSION: This study defines serum UA reference ranges for adolescents. It also reveals some intriguing relationships between UA and demographic and clinical characteristics that warrant further studies to examine the pathophysiological role of $U A$ in various disease processes.

\footnotetext{
n humans and higher primates, uric acid (UA) is the final oxidation product of purine metabolism and is excreted in urine. Elevated serum UA levels can result either exogenously from increased intake of purine-rich foods, or endogenously from increased production as seen in certain malignancies and inborn errors of metabolism; elevated serum UA levels can also result from decreased renal clearance. It is becoming apparent that UA homeostasis and its relationship with health and disease is complex. Elevated serum UA is associated with hypertension (HTN), as well as with diabetes, cardiovascular disease, endothelial dysfunction, obesity, metabolic syndrome, nephrolithiasis, intellectual disabilities, and all-cause mortality (1-8). On the other hand, some recent investigations provide evidence that UA may be beneficial as an antioxidant/freeradical scavenger in certain patient groups (9-11).
}

The association between elevated serum UA and HTN in both adults and children is well-described $(1,12)$. HTN is one of the most prevalent diseases in the Western world and tracking studies suggest that children and adolescents with elevated blood pressure (BP) are more likely to be hypertensive adults and develop metabolic syndrome $(13,14)$. Despite multiple investigations into the relationship between UA and HTN in children, little is known about the normal range of serum UA levels in children and adolescents. Previous studies on UA levels in children were either based on small sample size or presented only mean UA values (15-18). Further, given that previous studies were not representative of the US population, it is possible that they do not broadly represent recent trends and changes in diet and demographics.

Similarly, associations between serum UA levels and socioeconomic status, diet, and other demographic, clinical, and laboratory parameters have not been established in adolescents. Therefore, the aims of this study were to (i) examine serum UA distributions in a large, nationally representative cohort of US adolescents; (ii) examine the relationships between UA values and dietary and demographic variables; (iii) examine the relationships between $\mathrm{UA}$ values and BP and other cardiovascular risk factors; and (iv) examine the determinants of elevated UA levels in a multivariate regression model.

\section{RESULTS}

\section{Relationship Between Serum UA and Demographics}

A total of 1,912 adolescents in NHANES 2005-2008, 13-18 y of age and representing 19,888,299 adolescents, were included in these analyses (Figure 1). The mean ( \pm SD) UA level was $5.14 \pm 1.45 \mathrm{mg} / \mathrm{dl}$, and the levels were higher with increasing age and for the male gender as compared with the female gender. Participants categorized as belonging to the non-Hispanic white race had significantly higher mean UA levels $(5.22 \pm 0.99 \mathrm{mg} / \mathrm{dl})$ as compared with non-Hispanic blacks $(4.89 \pm 1.98 \mathrm{mg} / \mathrm{dl})$ and Mexican Americans $(5.03 \pm$ $2.33 \mathrm{mg} / \mathrm{dl}$ ), $P=0.002$ and 0.0164 , respectively (Table 1 ).

The relationships between weighted UA quartiles (estimated by quantile regression) and demographic and biochemical characteristics are shown in Table 2. An increase in UA

'Pediatric Nephrology and Hypertension, Medical University of South Carolina Children's Hospital, Charleston, South Carolina; ${ }^{2}$ Department of Surgery, Medical University of South Carolina, Charleston, South Carolina; ${ }^{3}$ Independent consultant in epidemiology, Los Gatos, California. Correspondence: Ibrahim F. Shatat (Shatat@musc.edu) 
quartile was significantly associated with older age, higher BMI, greater height, greater weight, male gender, and black race, and was inversely associated with higher urinary albu$\mathrm{min} /$ creatinine ratio (ACR) per weighted least squares analyses. Significantly more non-Hispanic white, male, and older

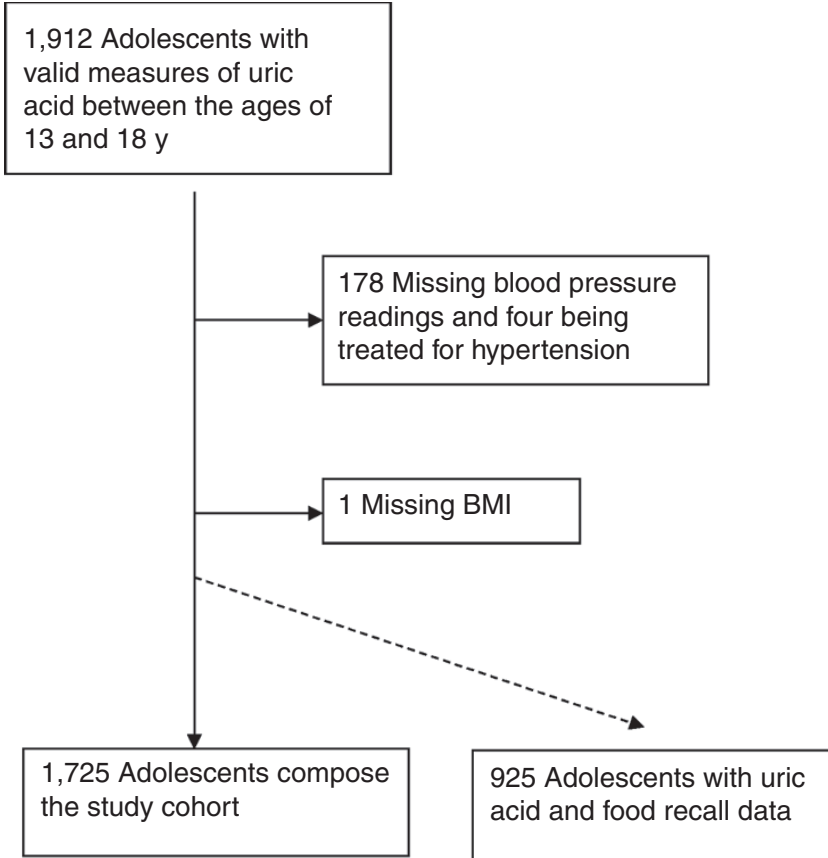

Figure 1. Flow chart of the study cohort. adolescents with higher BMI $z$-scores were in the higher UA quartiles (i.e., higher UA levels). There was no significant trend in the relationship between UA quartile and either cholesterol or estimated glomerular filtration rate. The lowest C-reactive protein levels were observed in the second quartile (UA 4.3$5.1 \mathrm{mg} / \mathrm{dl})(1 \mathrm{mg} / \mathrm{dl}=59.48 \mu \mathrm{mol} / \mathrm{l} \mathrm{SI}$ units$)$, but this trend was not statistically significant $(P=0.365)$.

\section{Relationship of UA Levels With Diet and With Poverty}

The association between the results of the dietary recall interviews (averaged over $2 \mathrm{~d}$ ) and UA quartiles is shown in Table 3. The relationships between dietary data and increasing UA quartile were "J" shaped for all dietary components, with the exception of fiber. The second quartile of UA (4.3$5.1 \mathrm{mg} / \mathrm{dl}$ ) was associated with the lowest percentage of poverty (Table 2).

\section{Relationship of UA With BP}

A total of 273 participants (15.8\%) had elevated BP, defined as $\geq 90$ th percentile for age, sex, and height. Of these, 50 (2.9\%) had HTN and 223 (12.9\%) had pre-HTN. UA levels were not significantly different between the prehypertensive and the hypertensive groups. Systolic BP value, and classification as HTN/pre-HTN were significantly associated with higher UA quartiles $(P=0.019$ and $<0.001$, respectively, Table 4$)$.

\section{Determinants of UA Levels}

In a weighted linear regression model, sex, older age, nonHispanic white race, and having a higher BMI $z$-score were

Table 1. Nationally representative percentiles of uric acid distribution in 13-18-y-old persons, NHANES 2005-2008

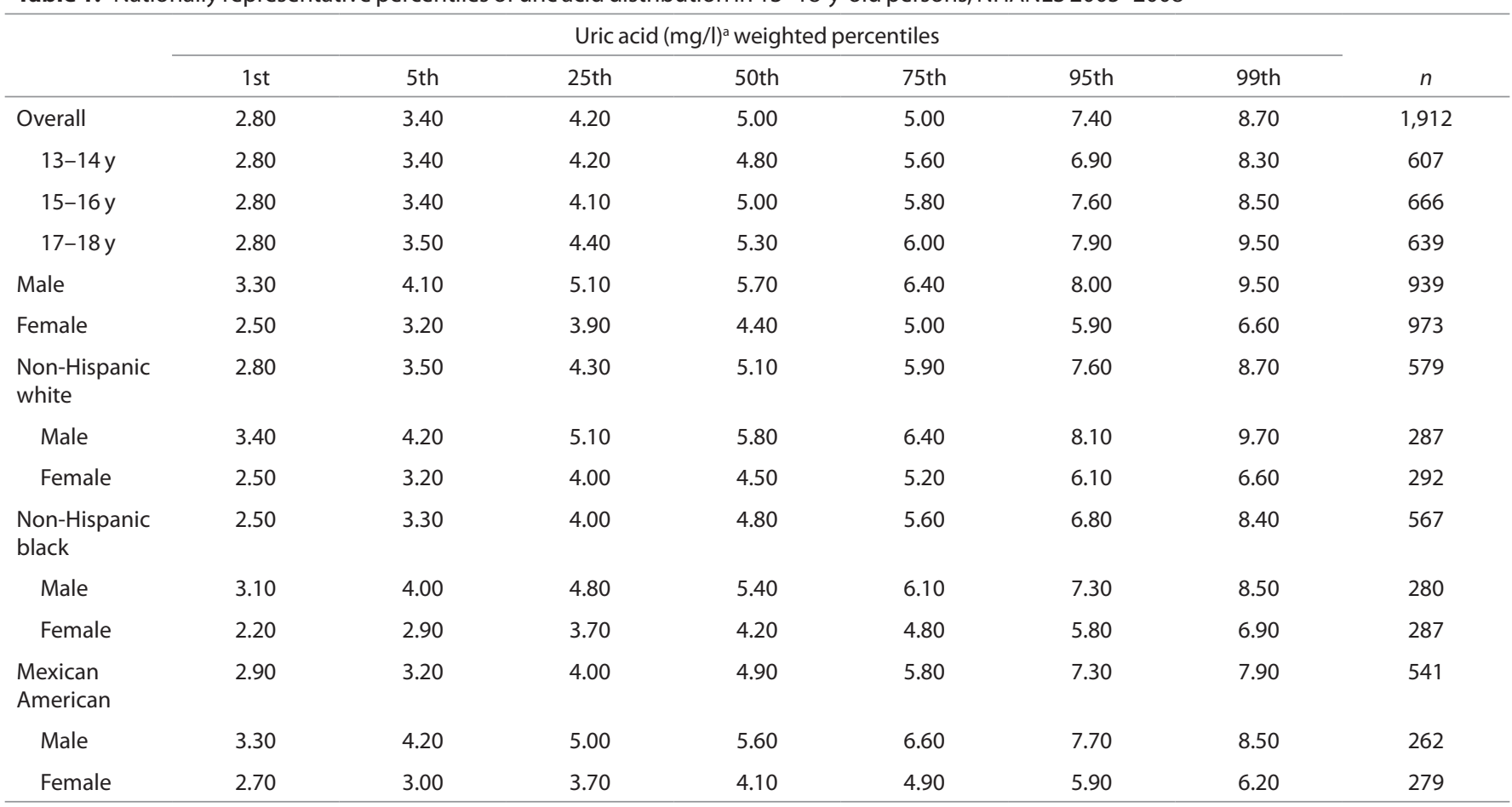


Table 2. Characteristics of persons aged 13-18 y by quartile of uric acid, NHANES 2005-2008

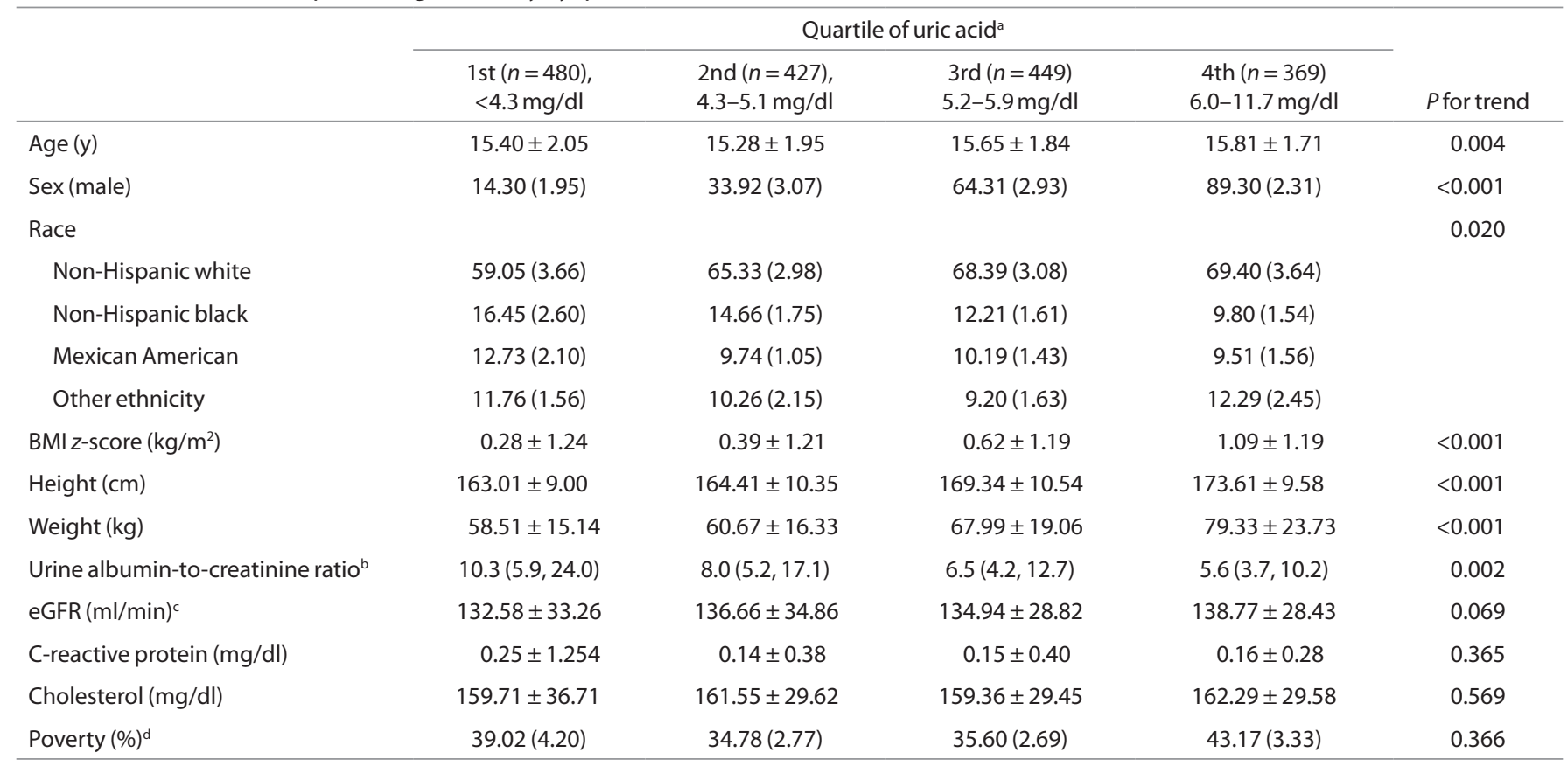

Continuous variables are presented as mean \pm SD; categorical variables are presented as proportion (SE). P for trend calculated by weighted least squares analyses. Percentages may not total $100 \%$ due to rounding.

NHANES, National Health and Nutrition Examination Survey.

aunweighted sample size is not equal in each quartile because quartiles of uric acid are based on a weighted distribution. ${ }^{\circ}$ Presented as median (interquartile range). Estimated glomerular filtration rate (eGFR) estimated using Schwartz formula (39). Available for 2005-2006 sample only ( $n=1,031)$. dPoverty-income ratio $<1.0$.

Table 3. Dietary characteristics of persons aged 13-18y, NHANES 2005-2008

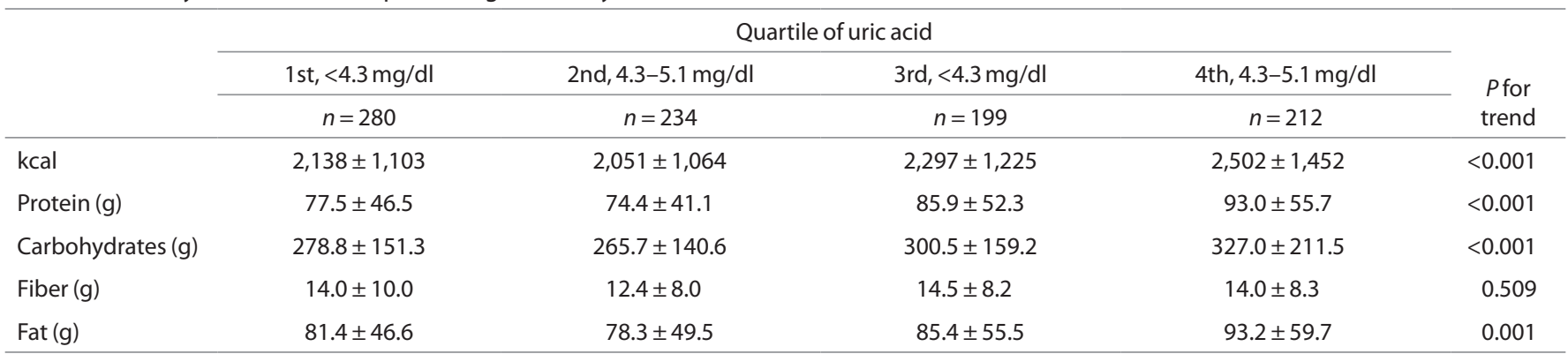

Dietary recall averaged over $2 \mathrm{~d}$. Presented as mean \pm SD. Weighted uric acid quartiles estimated by quantile regression. $P$ for trend calculated by weighted least squares analyses.

NHANES, National Health and Nutrition Examination Survey.

significantly associated with higher UA levels. None of the variables relating to nutritional intake were significantly associated with UA per analysis of data from a smaller, fully adjusted model using 2005-2006 data only (data not shown).

\section{DISCUSSION}

In this study, we defined percentiles of UA distribution in a nationally representative sample of adolescents 13-18 y of age. Furthermore, we examined the relationship of UA with dietary factors as well as with multiple demographic and clinical characteristics. We also examined independent determinants of UA levels.

There are no reference ranges of values for plasma UA in children and adolescents based on large population studies. Earlier studies reported a mean of $3.6 \mathrm{mg} / \mathrm{dl}$ (range 2.0-6.7) in
204 children aged 0-13 y hospitalized for a variety of illnesses (18). In a more recent single-center study using a different assay, UA levels were found to vary with age and sex, the ranges being $2.3-6.0 \mathrm{mg} / \mathrm{dl}$ and $3.5-8.0 \mathrm{mg} / \mathrm{dl}$ for $\geq 13$-y-old female and male participants, respectively (19). In the same study, the authors used adult values as reference intervals for female participants $\geq 13$ y of age and male participants $\geq 17$ y old.

In our cohort, UA levels followed a normal distribution. The mean UA level was $5.14 \pm 1.45 \mathrm{mg} / \mathrm{dl}$ (range 1st-99th percentile, $2.8-8.7 \mathrm{mg} / \mathrm{dl})$. UA levels were higher in male participants than in female participants; also, the levels increased with BMI in a linear fashion, consistent with recently published data by Lee et al. (20).

Age-related and race-related differences in serum UA levels have not been well described. In our cohort, white adolescents 
Table 4. Characteristics of blood pressure in persons aged 13-18 y by quartile of uric acid, NHANES 2005-2008

\begin{tabular}{lccccc}
\hline & \multicolumn{4}{c}{ Quartile of uric acid } \\
\cline { 2 - 4 } & 1st $(n=480),<4.3 \mathrm{mg} / \mathrm{dl}$ & 2nd $(n=427), 4.3-5.1 \mathrm{mg} / \mathrm{dl}$ & 3rd $(n=449), 5.2-5.9 \mathrm{mg} / \mathrm{dl}$ & 4 th $(n=369), 6.0-11.7 \mathrm{mg} / \mathrm{dl}$ & $\begin{array}{c}P \text { for } \\
\text { trend }\end{array}$ \\
\hline Systolic index & $0.84 \pm 0.09$ & $0.84 \pm 0.08$ & $0.84 \pm 0.08$ & $0.86 \pm 0.08$ & 0.019 \\
Diastolic index & $0.73 \pm 0.15$ & $0.74 \pm 0.14$ & $0.73 \pm 0.15$ & $0.71 \pm 0.14$ \\
HTN/pre-HTN (\%) ${ }^{\mathrm{a}}$ & $6.04(1.36)$ & $9.86(2.00)$ & $16.81(3.06)$ & 0.057 \\
\end{tabular}

Presented as mean \pm SD. Weighted uric acid quartiles estimated by quantile regression. $P$ for trend calculated by weighted least squares analyses.

NHANES, National Health and Nutrition Examination Survey.

aPrehypertension (pre-HTN) and hypertension (HTN) defined according to the fourth report on the diagnosis, evaluation, and treatment of high blood pressure in children and adolescents (ref. 35).

had significantly higher UA levels as compared with both black and Hispanic adolescents.

There is a relationship between socioeconomic status and obesity in both adults and children in the United States (21). In developing nations, children living in poverty generally experience true protein-energy malnutrition, whereas in developed nations poverty predisposes to obesity and micronutrient malnourishment (22). Although poverty and protein-energy malnutrition have been shown to lead to lower serum UA levels (23), no relationship between socioeconomic status and UA has previously been described in developed nations.

Our data show the highest UA levels to be associated with the highest percentage of children living in poverty, whereas UA levels in the range $4.3-5.1 \mathrm{mg} / \mathrm{dl}$ was associated with the lowest percentage of poverty. Also, our data reveal a J-shaped relationship between UA and the intake of all dietary components except fiber. Previous investigations regarding the association between dietary intake and UA showed increased serum UA levels with higher intake of sugar-sweetened beverages (24), calories (25), and protein $(25,26)$, and lower intake of vitamin C (27). A study using NHANES data in adults revealed that a higher intake of fiber correlated with lower serum UA levels (28), which is consistent with our findings. However, a small, prospective study in Norway showed no effect of increased fiber intake on serum UA levels in adults (29). The observed relationships suggest that dietary factors do relate to UA levels but do not solely explain higher or lower UA levels. Dietary factors did not remain as an independent determinant of UA levels in the final regression model (Table 5).

The prevalence of HTN in our cohort was $2.9 \%$, which is close to the value of $3.7 \%$ previously reported from older NHANES data by Din-Dzietham et al. $(30,31)$, but lower than the value of $4.5 \%$ reported by Sorof et al. in a population of children in a major metropolitan city (31). These differences are probably related to data from differing time periods as regards the former study, and differences in study population (including higher BMI) as regards the latter study.

Results of recent studies support the possible role of UA as a mediator of high BP $(1,2)$. In this study, we classified the cohort's BP values on the basis of age, height, and gender as normotensive, prehypertensive, and hypertensive. A total of 269 adolescents had an abnormal BP classification, which was significantly associated with the UA quartile trend, $P<$ 0.001. Our findings are consistent with those of Gruskin and colleagues, who compared adolescents having essential HTN to age-matched, healthy controls with normal BP and found that those with HTN higher serum UA levels (32). Feig and Johnson observed that the mean serum UA level in children with "white-coat HTN" was $3.6 \pm 0.7 \mathrm{mg} / \mathrm{dl}$; in those with secondary HTN it was higher $(4.3 \pm 1.4 \mathrm{mg} / \mathrm{dl}, P=0.008)$, and in children with primary HTN UA was significantly elevated (6.7 $\pm 1.3 \mathrm{mg} / \mathrm{dl}, P=0.001$ ) (2).

To our knowledge, there have been no previous large pediatric studies examining the relationship between UA and microalbuminuria. The inverse relationship observed in this cohort is possibly influenced by the inclusion of a poorly nourished group with lower UA levels (reflected by dietary intake data and higher C-reactive protein). Also, it is important to stress that the albuminuria levels in this cohort were below those that are considered to be clinically relevant in adult populations; the significance of microalbuminuria in children has not yet been elucidated.

Our finding that the highest C-reactive protein levels are in the lowest UA quartile group is intriguing and may reflect the presence of a possibly poorly nourished group in the lowest quartile. Of note, few previous studies demonstrated a "U-shaped" or a J-shaped relationship between various cardiovascular outcomes and UA levels $(33,34)$.

Given the lack of universally accepted upper normal limits for UA in adolescents, we built three statistical models to examine two different cutoff points considered as representing elevated UA (95\%, and UA levels in the upper weighted quartile of UA (data not shown)), and a third model to examine predictors of UA level considered as a continuous variable. The determinants of UA were similar in the three models, with gender, race, BMI z-score, and age representing independent determinants of UA level. Systolic BP, diastolic BP, BP classification, urinary ACR, dietary factors, and poverty-income ratio did not remain as independent determinants in the final model. It is possible that BP did not remain in the final model because of the relatively small number of adolescents with elevated BP in the cohort. An alternative possibility is that it did not remain in the final model because of its significant but weak correlation with BMI $z$-score.

Our study had some limitations. Although we were able to examine the relationship between UA levels and other variables, the nature of the study design did not allow for examination of causality. Another limitation of the study was the 
Table 5. Predictive model 3: continuous UA.

\begin{tabular}{lcr}
\hline Variable & Coefficient $(95 \% \mathrm{Cl})$ & $P$ value \\
\hline Sex $($ female $)$ & $-1.32(-1.43,-1.24)$ & $<0.001$ \\
Age $(y)$ & $0.08(0.05,0.12)$ & $<0.001$ \\
Race & Referent & \\
Non-Hispanic white & $-0.50(-0.64,-0.36)$ & $<0.001$ \\
Non-Hispanic black & $0.37(-0.54,-0.19)$ & $<0.001$ \\
Mexican American & $0.18(-0.32,0.17)$ & 0.570 \\
Other & $0.36(0.31,0.44)$ & $<0.001$ \\
BMl z-score & & \\
\hline
\end{tabular}

Coefficients and $P$ values estimated using weighted linear regression (least squares). $\mathrm{Cl}$, confidence interval; $\mathrm{UA}$, uric acid.

limited volume of dietary data collected from the NHANES 2005-2006 participants. In addition, the retrospective nature of our study, along with the reliance on dietary recall, instills inherent limitations as compared with a prospective study involving strict dietary control. Despite these limitations, our study, which used data from the largest nationally representative sample of adolescents for the purpose of defining reference ranges for serum UA, was also the first to examine the relationship between UA levels and dietary factors, poverty, and BP classification. It is also worth noting that the NHANES database involves the use of the same assay to measure UA levels, thereby eliminating differences in measurement methods frequently encountered in multicenter studies. It also used a standardized BP classification based on the fourth report on the diagnosis, evaluation, and treatment of high BP in children and adolescents (35). Also, conducting the study in a healthy, young cohort minimized confounding by comorbidities, which is a major weakness of existing smaller studies.

\section{Conclusions}

This work defines the frequency distribution of serum UA levels in a sample that is representative of the US adolescent population, and examines the relationship between UA levels and multiple demographic and biochemical characteristics as well as known cardiovascular risk determinants such as BP and diet. Sex, age, race, and BMI $z$-score were found to be independent determinants of higher UA levels. Further prospective studies are needed to address the relationship between UA levels and cardiovascular morbidities in pediatric patients.

\section{METHODS}

\section{Study Population}

Data from the NHANES for the years 2005-2008 were analyzed. NHANES is an ongoing nationally representative cross-sectional survey of the civilian, noninstitutionalized US population and uses a complex, stratified, multistage probability design. The survey is performed by the National Center for Health Statistics at the Centers for Diseases Control and Prevention, and the results are released in 2-y increments. Survey participants underwent standardized interviews, physical examinations, and laboratory testing in their homes and at a mobile examination center (MEC).

Serum UA measurements were obtained for all individuals $\geq 12$ $y$ of age in NHANES 2005-2008. Of the 2,125 children and adolescents $12-19$ y of age, valid measures for serum UA were available for $n=1,912$. In our study, analysis was limited to data relating to adolescents 13-19 y of age for whom serum UA measurements and complete data on other variables of interest were available $(n=1,725)$. Because dietary intake information is not reported in the public-access NHANES 2007-2008 data, the analysis of the association between UA and dietary intake was limited to adolescents from NHANES 2005$2006(n=925)$.

NHANES 2005-2008 was approved by the National Center for Health Statistics Institutional Review Board. All participants $\geq 18$ y of age provided informed consent; for those $<18 \mathrm{y}$ of age, parents/guardians provided informed consent.

\section{Study Variables}

The demographic variables included in our study were age, gender, and self-reported race/ethnicity (categorized as non-Hispanic black, non-Hispanic white, Mexican American, and other). Poverty-income ratio is the ratio of a family's income to the poverty threshold as defined by the US Census Bureau. In NHANES, a poverty-income ratio $\leq 1$ was defined as being below the poverty threshold.

Detailed information on dietary intake was collected from the 2005-2006 NHANES participants during the MEC. Food intake histories were used to estimate the types and amounts of foods and beverages consumed during the 24 -h period before the interview and to estimate intake of energy, nutrients, and other food components from those foods and beverages. All NHANES participants were eligible for two 24-h dietary recall interviews. The first dietary recall interview was collected in person during the MEC. The second interview was by telephone 3-10 d after the MEC. Average dietary intake was used in the analysis.

Physical measures were obtained in the MEC in accordance with standardized protocol (36). Using a mercury sphygmomanometer, three measurements of systolic and diastolic BP were obtained for each participant. The participants were classified as having HTN if this mean value was $\geq$ the 95 th percentile for age, sex, and height, and as having pre-HTN if the mean BP was $\geq$ the 90th percentile but $<$ the 95 th percentile (35). The BP index (mean BP divided by the 95th percentile of BP for age, gender, and height) was calculated for each participant. Each participant's height and weight were measured. BMI was calculated as weight in kilograms divided by height in meters squared. BMI $z$-scores were calculated based on 2000 Centers for Diseases Control and Prevention growth charts (37). Laboratory procedures are described in detail in the NHANES Laboratory/Medical Technologists Procedures Manual (38) Serum UA levels were measured using a colorimetric method. High-sensitivity C-reactive protein was measured using latexenhanced nephelometry. Serum cholesterol levels were measured on the Beckman Synchron LX20 (Beckman Coulter, Brea, CA). Urinary albumin levels were measured using a solid-phase fluorescent immunoassay. Urinary creatinine levels were measured using the Jaffe rate reaction with a CX3 analyzer (Beckman ASTRA, Brea, CA). The urinary ACR was calculated as urinary albumin value divided by urinary creatinine value. An ACR $\geq 30$ was used to define albuminuria. Serum creatinine was measured by means of the modified kinetic Jaffe reaction. Because differences were noted in serum creatinine values from the 1999-2000 survey when compared with a "gold standard," a correction factor was applied to adjust for errors in measurement. Glomerular filtration rate was estimated using the Schwartz formula (39).

\section{Statistical Analysis}

The data were analyzed using Stata (SE 10.1; Stata, College Station, TX). Standard errors for all estimates were obtained using the Taylorlinearized variance estimation. Four-y sample weights were used to account for the complex sampling design of NHANES, including unequal probabilities of selection, oversampling, and nonresponse. A $P$ value $<0.05$ was considered statistically significant.

Mean values for continuous variables and proportions for categorical variables were estimated on the basis of weighted UA quartiles. Statistical significance of linear trends across patient characteristics was determined by linear and logistic regression for continuous and 
categorical variables, respectively. Weighted percentiles of the distribution of UA were estimated using quantile regression, utilizing analytical weights. Weighted linear regression models (least squares) were utilized to examine the associations between UA and demographic, socioeconomic, and dietary risk factors.

The relationship between BP and UA was first examined using the frequency distribution of UA levels based on BP classification. Next, the relationship between BP and weighted UA quartiles and the relationship between BP index and UA levels were examined.

\section{STATEMENT OF FINANCIAL SUPPORT}

No financial assistance was received to support this study.

\section{REFERENCES}

1. Alper AB Jr, Chen W, Yau L, Srinivasan SR, Berenson GS, Hamm LL. Childhood uric acid predicts adult blood pressure: the Bogalusa Heart Study. Hypertension 2005;45:34-8.

2. Feig DI, Johnson RJ. Hyperuricemia in childhood primary hypertension. Hypertension 2003;42:247-52.

3. Khosla UM, Zharikov S, Finch JL, et al. Hyperuricemia induces endothelial dysfunction. Kidney Int 2005;67:1739-42.

4. Kramer CK, von Mühlen D, Jassal SK, Barrett-Connor E. A prospective study of uric acid by glucose tolerance status and survival: the Rancho Bernardo Study. J Intern Med 2010;267:561-6.

5. Wasserman A, Shnell M, Boursi B, Guzner-Gur H. Prognostic significance of serum uric acid in patients admitted to the Department of Medicine. Am J Med Sci 2010;339:15-21.

6. Lin JD, Lin PY, Lin LP, et al. Serum uric acid, hyperuricemia and body mass index in children and adolescents with intellectual disabilities. Res Dev Disabil 2009;30:1481-9.

7. Negri AL, Spivacow R, Del Valle E, et al. Clinical and biochemical profile of patients with "pure" uric acid nephrolithiasis compared with "pure" calcium oxalate stone formers. Urol Res 2007;35:247-51.

8. Ford ES, Li C, Cook S, Choi HK. Serum concentrations of uric acid and the metabolic syndrome among US children and adolescents. Circulation 2007;115:2526-32.

9. Ames BN, Cathcart R, Schwiers E, Hochstein P. Uric acid provides an antioxidant defense in humans against oxidant- and radical-caused aging and cancer: a hypothesis. Proc Natl Acad Sci USA 1981;78: 6858-62.

10. Waring WS, McKnight JA, Webb DJ, Maxwell SR. Uric acid restores endothelial function in patients with type 1 diabetes and regular smokers. Diabetes 2006;55:3127-32.

11. Amaro S, Urra X, Gómez-Choco M, et al. Uric acid levels are relevant in patients with stroke treated with thrombolysis. Stroke 2011;42:Suppl $1:$ S28-32.

12. Agamah ES, Srinivasan SR, Webber LS, Berenson GS. Serum uric acid and its relation to cardiovascular disease risk factors in children and young adults from a biracial community: the Bogalusa Heart Study. J Lab Clin Med 1991;118:241-9.

13. Chen X, Wang Y. Tracking of blood pressure from childhood to adulthood: a systematic review and meta-regression analysis. Circulation 2008;117:3171-80.

14. Sun SS, Grave GD, Siervogel RM, Pickoff AA, Arslanian SS, Daniels SR. Systolic blood pressure in childhood predicts hypertension and metabolic syndrome later in life. Pediatrics 2007;119:237-46.

15. Ghoshal AK, Soldin SJ. Evaluation of the Dade Behring Dimension RxL: integrated chemistry system-pediatric reference ranges. Clin Chim Acta 2003;331:135-46.

16. Invitti C, Gilardini L, Viberti G. Obesity and the metabolic syndrome in children and adolescents. N Engl J Med 2004;351:1146-8; author reply $1146-8$.

17. Lockitch G, Halstead AC, Albersheim S, MacCallum C, Quigley G. Age- and sex-specific pediatric reference intervals for biochemistry analytes as measured with the Ektachem-700 analyzer. Clin Chem $1988 ; 34: 1622-5$.
18. Harkness RA, Nicol AD. Plasma uric acid levels in children. Arch Dis Child 1969;44:773-8.

19. Burritt MF, Slockbower JM, Forsman RW, Offord KP, Bergstralh EJ, Smithson WA. Pediatric reference intervals for 19 biologic variables in healthy children. Mayo Clin Proc 1990;65:329-36.

20. Lee MS, Wahlqvist ML, Yu HL, Pan WH. Hyperuricemia and metabolic syndrome in Taiwanese children. Asia Pac J Clin Nutr 2007:16 Suppl 2:594-600.

21. Wang Y, Beydoun MA. The obesity epidemic in the United Statesgender, age, socioeconomic, racial/ethnic, and geographic characteristics: a systematic review and meta-regression analysis. Epidemiol Rev 2007;29:6-28.

22. Tanumihardjo SA, Anderson C, Kaufer-Horwitz M, et al. Poverty, obesity, and malnutrition: an international perspective recognizing the paradox. J Am Diet Assoc 2007;107:1966-72.

23. Etukudo MH, Agbedana EO, Akinyinka OO, Osifo BO. Plasma electrolytes, total cholesterol, liver enzymes, and selected antioxidant status in protein energy malnutrition. Afr J Med Med Sci 1999;28:81-5.

24. Nguyen S, Choi HK, Lustig RH, Hsu CY. Sugar-sweetened beverages, serum uric acid, and blood pressure in adolescents. J Pediatr 2009;154:807-13.

25. Dessein PH, Shipton EA, Stanwix AE, Joffe BI, Ramokgadi J. Beneficial effects of weight loss associated with moderate calorie/carbohydrate restriction, and increased proportional intake of protein and unsaturated fat on serum urate and lipoprotein levels in gout: a pilot study. Ann Rheum Dis 2000;59:539-43.

26. Frank H, Graf J, Graf J, et al. Effect of short-term high-protein compared with normal-protein diets on renal hemodynamics and associated variables in healthy young men. Am J Clin Nutr 2009;90:1509-16.

27. Gao X, Curhan G, Forman JP, Ascherio A, Choi HK. Vitamin C intake and serum uric acid concentration in men. J Rheumatol 2008;35:1853-8.

28. Sun SZ, Flickinger BD, Williamson-Hughes PS, Empie MW. Lack of association between dietary fructose and hyperuricemia risk in adults. Nutr Metab (Lond) 2010;7:16.

29. Birketvedt GS, Aaseth J, Florholmen JR, Ryttig K. Long-term effect of fibre supplement and reduced energy intake on body weight and blood lipids in overweight subjects. Acta Medica (Hradec Kralove) 2000;43:129-32.

30. Din-Dzietham R, Liu Y, Bielo MV, Shamsa F. High blood pressure trends in children and adolescents in national surveys, 1963 to 2002. Circulation 2007;116:1488-96.

31. Sorof JM, Lai D, Turner J, Poffenbarger T, Portman RJ. Overweight, ethnicity, and the prevalence of hypertension in school-aged children. Pediatrics 2004;113(3 Pt 1):475-82.

32. Gruskin AB. The adolescent with essential hypertension. Am J Kidney Dis 1985;6:86-90.

33. Hsu SP, Pai MF, Peng YS, Chiang CK, Ho TI, Hung KY. Serum uric acid levels show a 'J-shaped' association with all-cause mortality in haemodialysis patients. Nephrol Dial Transplant 2004;19:457-62.

34. Seet RC, Kasiman K, Gruber J, et al. Is uric acid protective or deleterious in acute ischemic stroke? A prospective cohort study. Atherosclerosis 2010;209:215-9.

35. National High Blood Pressure Education Program Working Group on High Blood Pressure in Children and Adolescents. The fourth report on the diagnosis, evaluation, and treatment of high blood pressure in children and adolescents. Pediatrics 2004;114:555-76.

36. National Health and Nutrition Examination Survey. Physician examination procedures manual, 2000. <http://www.cdc.gov/nchs/data/nhanes/ nhanes_03_04/PE.pdf>.

37. Kuczmarski RJ, Ogden CL, Guo SS, et al. 2000 CDC Growth Charts for the United States: methods and development. Vital Health Stat 11 2002:1-190.

38. National Health and Nutrition Examination Survey. Laboratory procedures manual, 2001. <http://www.cdc.gov/nchs/data/nhanes/nhanes_05_06/ lab_d_generaldoc.pdf $>$.

39. Schwartz GJ, Muñoz A, Schneider MF, et al. New equations to estimate GFR in children with CKD. J Am Soc Nephrol 2009;20:629-37. 